\title{
The combination therapy of transarterial chemoembolisation and sorafenib is the preferred palliative treatment for advanced hepatocellular carcinoma patients: a meta- analysis
}

Zhoujing Cheng ${ }^{1+}$, Lin He ${ }^{2 \dagger}$, Yingjie Guo ${ }^{3}$, Yuhua Song ${ }^{2}$, Shasha Song ${ }^{1}$ and Lijiu Zhang ${ }^{1 *}$ (i)

\begin{abstract}
Background: To compare the efficacy of three types of palliative therapy for advanced hepatocellular carcinoma (HCC), including transarterial chemoembolisation (TACE) monotherapy, sorafenib alone and their combination.

Methods: The databases of PubMed, Embase and Cochrane Library were retrieved. The odds ratio (OR) with its 95\% confidence interval $(\mathrm{Cl})$ was used to investigate the binary variables, and the standardised mean difference (SMD) with its $95 \% \mathrm{Cl}$ was employed to evaluate the continuous variables. All statistical tests were performed by using Stata/SE, version 12.0.

Results: Thirty-one clinical studies, containing 5125 unique cases of patients with advanced HCC, were included. There were significant improvements in overall survival (OS) (pooled SMD $=2.54 ; 95 \% \mathrm{Cl} 1.74-3.34$ ) and time to progression (TTP) (pooled SMD $=2.49 ; 95 \% \mathrm{Cl} 0.87-4.12$ ) of the patients after receiving the combination therapy of TACE and sorafenib, compared to TACE monotherapy, and the OS in the combined treatment cohort was also longer than that in the sorafenib-alone cohort (pooled SMD $=2.92 ; 95 \% \mathrm{Cl} 1.72-4.13$ ). The combination therapy group in comparison to the TACE group benefited a significantly increased overall response rate (ORR) (pooled OR $=2.61$; $95 \% \mathrm{Cl} 1.43-4.77$ ), 1-year (pooled $\mathrm{OR}=2.96$; 95\% Cl 1.71-5.14) and 2-year (pooled $\mathrm{OR}=1.64$; $95 \% \mathrm{Cl} 1.18-$ 2.28) survival rates and reduced disease progression rate (DPR) (pooled $\mathrm{OR}=0.47 ; 95 \% \mathrm{Cl} 0.33-0.68$ ); in parallel, the ORR in the group was also significantly higher than that in the sorafenib-alone group (pooled $\mathrm{OR}=3.62 ; 95 \% \mathrm{Cl}$ 1.28-10.22), although without a difference in the DPR (pooled $\mathrm{OR}=0.28 ; 95 \% \mathrm{Cl} 0.05-1.48$ ). In addition, we discovered that the 1-year (pooled $\mathrm{OR}=1.39 ; 95 \% \mathrm{Cl} 0.84-2.29$ ) and 2-year (pooled $\mathrm{OR}=1.70 ; 95 \% \mathrm{Cl} 0.69-4.18$ ) survival rates in the TACE monotherapy cohort were not significantly different to those in the sorafenib-alone cohort.

(Continued on next page)
\end{abstract}

\footnotetext{
* Correspondence: zhanglijiu6336@163.com

${ }^{\dagger}$ Zhoujing Cheng and Lin He contributed equally to this work.

'Department of Gastroenterology, The Second Hospital of Anhui Medical University, No.678 Furong Road, Jingkai District, Hefei, Anhui Province, China Full list of author information is available at the end of the article
}

C C The Author(s). 2020 Open Access This article is licensed under a Creative Commons Attribution 4.0 International License, which permits use, sharing, adaptation, distribution and reproduction in any medium or format, as long as you give appropriate credit to the original author(s) and the source, provide a link to the Creative Commons licence, and indicate if changes were made. The images or other third party material in this article are included in the article's Creative Commons licence, unless indicated otherwise in a credit line to the material. If material is not included in the article's Creative Commons licence and your intended use is not permitted by statutory regulation or exceeds the permitted use, you will need to obtain permission directly from the copyright holder. To view a copy of this licence, visit http://creativecommons.org/licenses/by/4.0/. The Creative Commons Public Domain Dedication waiver (http://creativecommons.org/publicdomain/zero/1.0/) applies to the data made available in this article, unless otherwise stated in a credit line to the data. 
(Continued from previous page)

Conclusion: The combination therapy is more effective than monotherapy in improving the prognostic outcomes of patients with advanced HCC. Therefore, we recommend it as the preferred treatment intervention for those patients.

Keywords: TACE, Sorafenib, Hepatocellular carcinoma, Meta-analysis

\section{Background}

Hepatocellular carcinoma (HCC) is one of the most common gastrointestinal malignancies and the third most common cause of cancer-related death, with an approximate proportion of $90 \%$ in primary malignant liver tumours in adults $[1,2]$. The most effective way to treat a tumour in HCC is surgically, but only less than $18 \%$ of patients undergo it [3]; many patients are deprived of the surgery opportunity when they are initially diagnosed with an advanced-stage disease. For patients with Barcelona Clinic Liver Cancer (BCLC) stage B or C HCC who are not eligible for surgery [4], it is recommended to receive transarterial chemoembolisation (TACE) or sorafenib as the treatment modality.

The implementation of TACE is mainly constituted of two steps: (1) the embolisation of the tumour-supplying arteries to induce tumour hypoxia and necrosis and (2) the delivery of a high concentration of cytotoxic chemotherapy medications through those arteries to reinforce the tumour necrosis [5]. However, the level of vascular endothelial growth factor (VEGF) increases after TACE $[6,7]$, which is considered a partial facilitator of tumour progression and metastasis [8].

Sorafenib is an oral multikinase inhibitor and has the ability to inhibit tumour cell proliferation and angiogenesis [9] by suppressing the VEGF signal pathway by inhibiting VEGF receptors [10]. Some phase III, randomised, placebo-controlled trials have demonstrated its efficacy in treating advanced $\mathrm{HCC}$, significantly prolonging the time to progression (TTP) and overall survival (OS) [9, 11]. Considering that sorafenib can inhibit VEGF signalling, it may be effective to reduce TACE-induced overproduction of VEGF, hence further ameliorating the disease control of advanced HCC after TACE. As expected, the results of many studies have indicated that patients with this carcinoma derived more survival benefits from the combination of sorafenib and TACE than from TACE alone [12-14].

To understand the effectiveness of TACE, sorafenib and their combination in the treatment of advanced HCC patients comprehensively, this meta-analysis, with a massive number of cases, aimed to collect all relevant data to compare the TTP, OS, disease progression rate (DPR), survival rate and overall response rate (ORR) of patients after different alleviative treatments.

\section{Methods}

\section{Search strategy}

The PubMed, Cochrane Library and Embase databases were electronically searched with the following retrieval strategy, in light of the Preferred Reporting Items for Systematic Review and Meta-Analysis (individual participant data) (PRISMA-IPD) statement [15]: (("Liver Neoplasms" [MeSH]) OR (Neoplasms, Hepatic) OR (Neoplasms, Liver) OR (Liver Neoplasm) OR (Neoplasm, Liver) OR (Hepatic Neoplasms) OR (Hepatic Neoplasm) OR (Neoplasm, Hepatic) OR (Cancer of Liver) OR (Hepatocellular Cancer) OR (Cancers, Hepatocellular) OR (Hepatocellular Cancers) OR (Hepatic Cancer) OR (Cancer, Hepatic) OR (Cancers, Hepatic) OR (Hepatic Cancers) OR (Liver Cancer) OR (Cancer, Liver) OR (Cancers, Liver) OR (Liver Cancers) OR (Cancer of the Liver) OR (Cancer, Hepatocellular) OR ((Liver OR Hepatic OR Hepatocellular) AND (Tumour OR Cancer OR Tumour OR Carcinoma OR Neoplasm)) OR (Cholangiocellular carcinoma) OR Cholangiocarcinoma OR HCCCC OR (combined HCC-CC) OR CHC OR (Mixed hepatocellular and cholangiocarcinoma)) AND ((TACE OR (Transcatheter arterial chemoembolisation) OR (Transcatheter hepatic arterial chemoembolisation) OR (Transarterial chemoembolisation)) AND Sorafenib) AND (Survival OR Response OR ORR OR OS OR (Overall survival) OR TTP OR (Time-to-progression) OR Progression). There were no restrictions during the retrieval process. The due date of citation searching was April 20, 2019.

\section{Inclusion criteria}

- Clinical trials published in English;

- Patients with advanced HCC;

- Publication recorded the prognoses of at least two treatment methods; and

- The prognoses at least included more than one of the following components: OS, TTP, ORR, DPR, 1year survival rate and 2-year survival rate. OS referred to the duration from the date of diagnosis to the date of death or lost to follow-up. TTP was defined as the time from randomisation to the appearance of radiologic evidence of disease progression. ORR was evaluated by enhanced computed tomography or magnetic resonance imaging before and 
after treatment. The assessment criteria of tumour progression and tumour response were both according to Response Evaluation Criteria in Solid Tumours (RECIST) version 1.1 or modified RECIST (mRECIST)

\section{Exclusion criteria}

- Non-English publication

- Single-arm study

- Article type: review, case report, study protocol and conference paper

- Other details that did not meet the inclusion criteria

The titles and abstracts of all citations were screened by two co-authors independently. They further respectively perused the full texts of potential studies and retained only the satisfactory ones that met the inclusion criteria. Any inconsistencies were resolved by discussion.

\section{Data abstraction}

Two co-authors used Microsoft Excel version 2016 (Microsoft Corporation, Redmond, WA, USA) to abstract the following information from all eligible studies: first author, publication year, study type, original nation, prognostic endpoint, number of analysed cases, median follow-up, frequency of tumour assessment, median age, drugs administrated in TACE and the initial sorafenib administration. If any disagreements existed, they were resolved by the third co-author.

\section{Statistical analysis}

The comparison of continuous variables involving TTP and OS was assessed by standardised mean difference (SMD) with its 95\% confidence interval (CI). Moreover, the crude odds ratio (OR) with its 95\% CI was used to evaluate the comparison of ORR, DPR and 1-year and 2year survival rates between different treatment interventions. The heterogeneity across included studies was detected by heterogeneity chi-squared test with its significance level of $P<0.1$ [16]. If the heterogeneity test was not statistically significant, the data was pooled by a fixed-effects inverse variance model; otherwise, a random-effects inverse variance model was used [16]. Egger's test, with its significance level of $P<0.05$, was used to detect the publication bias in all analyses, and a tool presented by Jadad and colleagues was applied to evaluate the quality of all randomised controlled trials (RCTs) (eTable 1 in Supplementary, page 1) [17]. All statistical tests were performed with Stata/SE software, version 12.0 .

\section{Results}

\section{Search results}

One thousand four hundred thirty-two potential citations were identified after systematic retrieval in the aforementioned databases. After the removal of duplicate citations $(N=269)$ and those types of work classified as review $(N=75)$, case report $(N=24)$ and conference paper $(N=465), 599$ records were qualified for title and abstract screening; 211 of them were excluded by this process, leaving 59 articles for full-text evaluation. Of those, 28 were omitted for lack of useful data $(N=3)$, non-English publication $(N=16)$, singlearm study $(N=8)$ and study protocol $(N=1)$. Ultimately, 31 eligible trials $[12,14,18-46]$ with 5125 unique patients with advanced HCC met the inclusion criteria. The PRISMA flow diagram of study selection is outlined in Fig. 1.

\section{Characteristics of included studies}

Table 1 provides the details of the 31 included studies, and Table 2 summarises the characteristics of these studies in the "patient-level" analysis. Of those, six (19.4\%) were RCTs that included a total of 1128 cases; $18(58.1 \%)$ originated in China, and 15 (48.4\%) applied 3-8-week frequency of tumour assessment, and the predominant treatment scenario was administration of 400 mg sorafenib orally twice a day. We also summarised other details in Tables 1 and 2, such as the publication year, median follow-up, median age in each treatment strategy, the primary endpoint and the chemotherapy drugs used in TACE.

\section{Time to progression and overall survival}

Five studies, containing 750 cases, were included in the analysis comparing the TTP with combination therapy to that of TACE; the pooled data showed that the TTP in patients with advanced HCC receiving combination therapy was significantly longer than that of those receiving TACE treatment alone (pooled SMD $=2.49 ; 95 \%$ CI 0.87-4.12) (Fig. 2a). Twelve clinical trials with 1984 cases and five available studies with 887 cases were respectively involved in the comparison of OS between combination therapy and TACE and that between combined therapy and sorafenib monotherapy. As presented in Fig. $2 \mathrm{~b}$ and c, the combination therapy significantly prolonged the OS of patients compared to the monotherapy of TACE (pooled SMD $=2.54 ; 95 \%$ CI 1.74-3.34) or sorafenib (pooled SMD $=2.92 ; 95 \%$ CI 1.72-4.13).

\section{Disease progression rate}

We obtained 15 and four articles, respectively, to compare the DPR under combined treatment to TACE and that of combination therapy to sorafenib. The pooled 


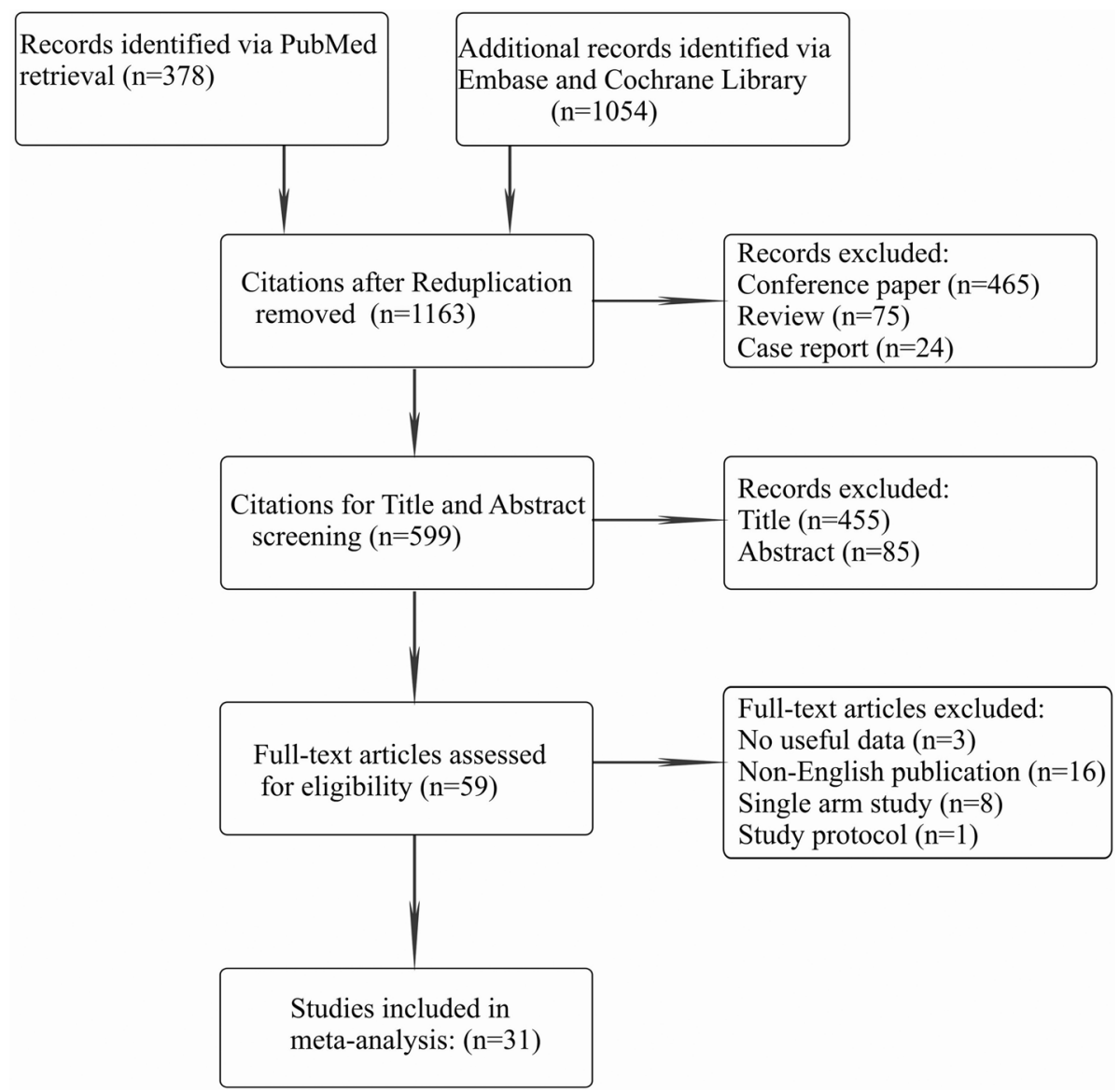

Fig. 1 PRISMA flow diagram of the study selection

data indicated that patients with advanced HCC undergoing combination therapy had a significantly lower DPR than those who received monotherapy of TACE (pooled OR $=0.47 ; 95 \%$ CI $0.33-0.68$ ) but not than those who took sorafenib alone orally (pooled OR = 0.28; 95\% CI 0.05-1.48) (Fig. 3a, b).

\section{One-year and 2-year survival rates}

As shown in Fig. 4a and b, seven and four useful studies, respectively, were included to assess the 1-year and 2year survival rates between combined treatment and TACE. Generally, patients in the combination therapy cohort benefited from significantly greater 1-year (pooled OR $=2.96 ; 95 \%$ CI 1.71-5.14) and 2-year (pooled OR $=1.64 ; 95 \%$ CI 1.18-2.28) survival rates than those in the TACE monotherapy cohort. We further analysed the survival rates of patients who received monotherapy with TACE compared to sorafenib, finding no significant difference of 1-year (pooled $\mathrm{OR}=1.39$; 95\% CI 0.84-2.29) and 2-year (pooled OR $=1.70$; $95 \%$ CI 0.69-4.18) survival rates between them (eFigure 1 in Supplementary page 1).

\section{Overall response rate}

Eight and four studies, respectively, were involved in the comparison of ORR between combination therapy and TACE and that between combination therapy and sorafenib. The results of the analysis suggested that patients exhibited a significantly increased ORR after receiving combination therapy, compared to those who underwent monotherapy with TACE (pooled OR $=2.61 ; 95 \% \mathrm{CI}$ $1.43-4.77$ ) or sorafenib (pooled $\mathrm{OR}=3.62 ; 95 \% \mathrm{CI}$ 1.28-10.22) (Fig. 5a, b).

\section{Publication bias}

The publication bias tests in most analyses were devoid of statistical significance, indicating no occurrence of publication bias among the studies involved in them; however, the analyses of DPR, 1-year survival rate and ORR after combination therapy versus TACE manifested discernible publication bias $(P=0.04,0.00$ and 0.01 , respectively) (eTable 1 on Supplementary page 2).

\section{Discussion}

Universally, patients with advanced HCC suffered from a poor prognosis due to the lack of surgical resection 


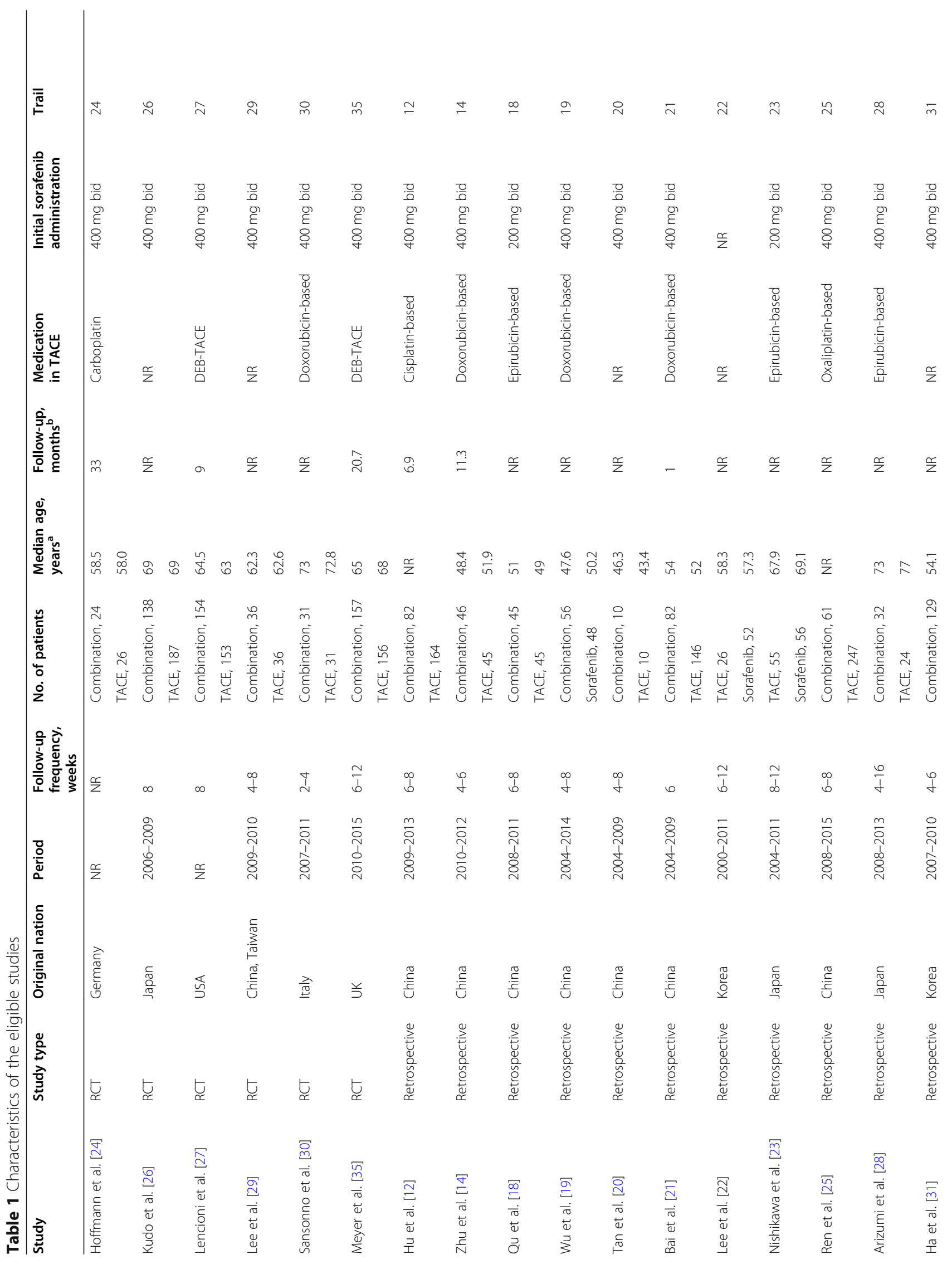




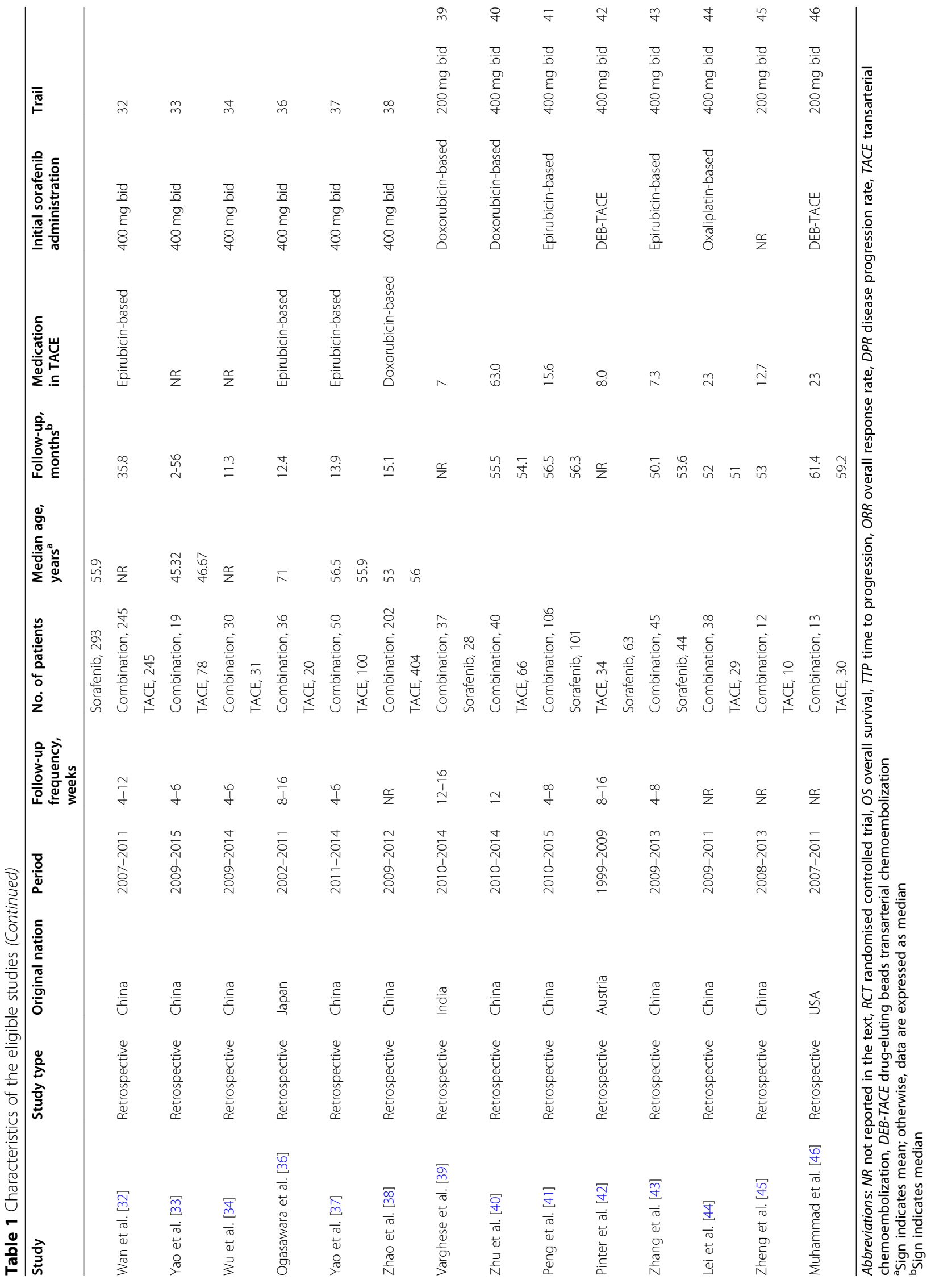


Table 2 Summary of the characteristics of the 31 included studies in the "patient-level" analysis

\begin{tabular}{|c|c|c|}
\hline Characteristic & Studies, no. $(\%)(N=31)$ & Advanced HCC patients, no. $(\%)(N=5125)$ \\
\hline \multicolumn{3}{|l|}{ Study type } \\
\hline $\mathrm{RCT}$ & $6(19.4)$ & $1128(22.0)$ \\
\hline Retrospective & $25(80.6)$ & $3997(78.0)$ \\
\hline Publication year, median (range) & 2016 (2010-2019) & - \\
\hline Follow-up, median (range), months & $14.45(5.4-63)$ & - \\
\hline \multicolumn{3}{|l|}{ Median age, median (range), years } \\
\hline Combination & $56(45.3-74)$ & - \\
\hline TACE & $57.5(43.4-74)$ & - \\
\hline Sorafenib & $56.3(50.2-74)$ & - \\
\hline \multicolumn{3}{|l|}{ Original nation } \\
\hline China & $18(58.1)$ & $3082(60.1)$ \\
\hline USA & $2(6.5)$ & $350(6.8)$ \\
\hline Japan & $4(12.9)$ & $547(10.7)$ \\
\hline Korea & $2(6.5)$ & $500(9.8)$ \\
\hline Germany & $1(3.2)$ & $50(1.0)$ \\
\hline Italy & $1(3.2)$ & $62(1.2)$ \\
\hline India & $1(3.2)$ & $124(2.4)$ \\
\hline UK & $1(3.2)$ & $313(6.1)$ \\
\hline Austria & $1(3.2)$ & $97(1.9)$ \\
\hline \multicolumn{3}{|l|}{ Primary endpoint } \\
\hline Overall survival & $18(58.1)$ & $2431(47.4)$ \\
\hline Time-to-progression & $4(12.9)$ & $743(14.5)$ \\
\hline Overall response rate & $9(29.0)$ & $1508(29.4)$ \\
\hline \multicolumn{3}{|l|}{ Follow-up frequency } \\
\hline 3-8 weeks & $15(48.4)$ & $2384(46.5)$ \\
\hline 8-12 weeks & $3(9.7)$ & $407(7.9)$ \\
\hline$\geq 12$ weeks & $8(25.8)$ & $1249(24.4)$ \\
\hline Not assessed & $5(16.1)$ & $1085(21.2)$ \\
\hline \multicolumn{3}{|l|}{ Medication in TACE } \\
\hline Doxorubicin-based & $7(22.6)$ & $1339(26.1)$ \\
\hline Epirubicin-based & $8(25.8)$ & $856(16.7)$ \\
\hline DEB-TACE ${ }^{\mathrm{b}}$ & $4(12.9)$ & $760(14.8)$ \\
\hline Others & $4(12.9)$ & $1111(21.7)$ \\
\hline Not assessed & $8(25.8)$ & $1059(20.7)$ \\
\hline \multicolumn{3}{|l|}{ Initial sorafenib administration } \\
\hline $200 \mathrm{mg}$ bid & $5(16.1)$ & $400(7.8)$ \\
\hline $400 \mathrm{mg}$ bid & $25(80.6)$ & $4647(90.7)$ \\
\hline Not applicable & $1(3.2)$ & $78(1.5)$ \\
\hline
\end{tabular}

Abbreviations: $H C C$ hepatocellular carcinoma, $R C T$ randomised controlled trial, TACE transarterial chemoembolization

${ }^{\mathrm{b}} \mathrm{TACE}$ with drug-eluting beads is performed with doxorubicin-loaded beads

opportunity and sustained their survival only with some palliative treatments. This meta-analysis proves that the combination therapy of TACE and sorafenib provides more advantages to improve ORR and prolong OS than monotherapy with either in treating these patients and enhances the increased 1-year and 2-year survival rates, lengthens TTP and decreases DPR, compared to TACE alone. We further found equivalent 1-year and 2-year survival rates between those who received TACE and only orally took sorafenib. 


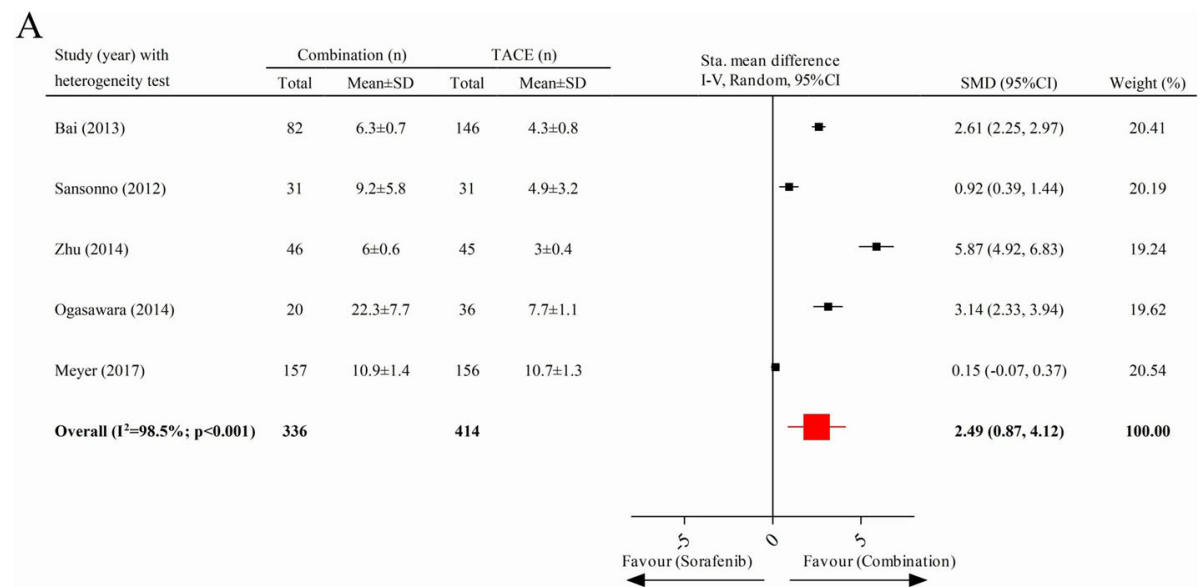

B

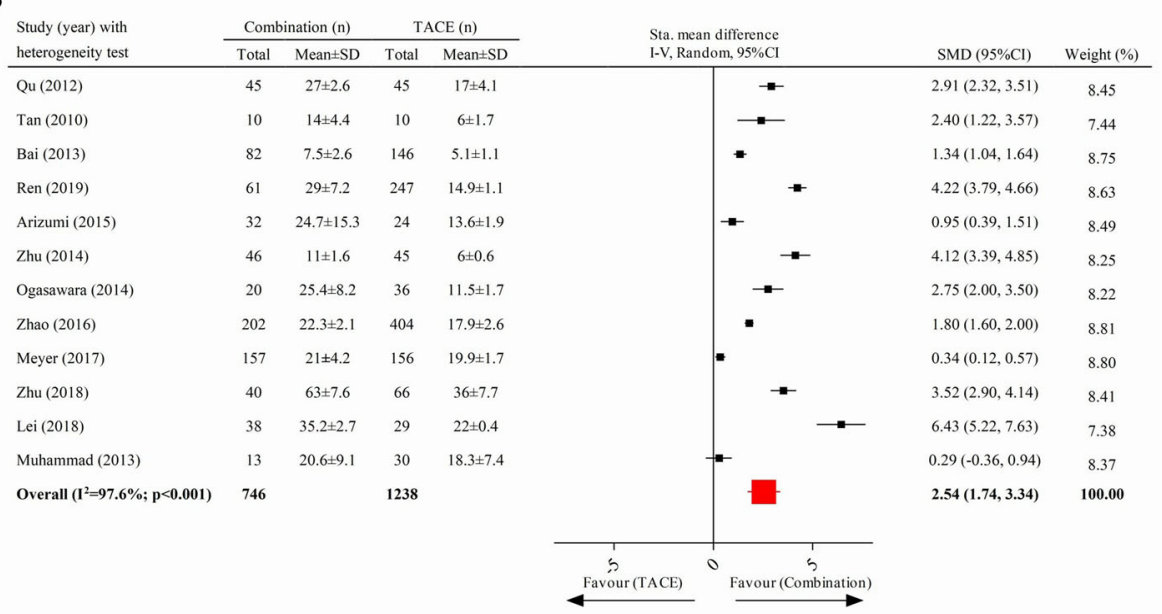

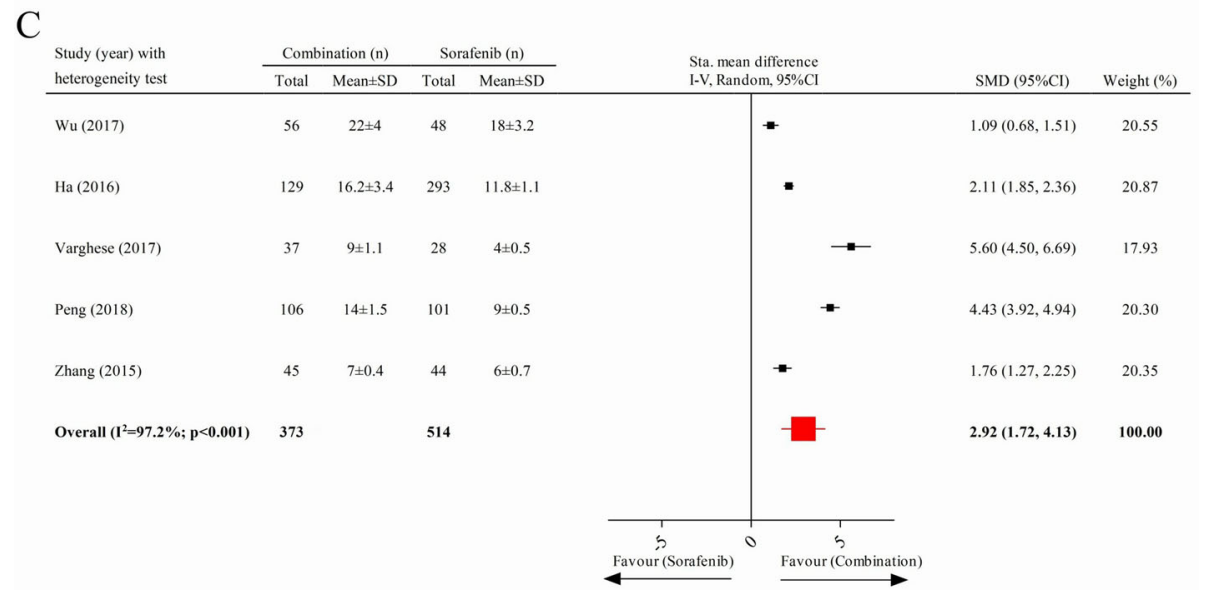

Fig. 2 Comparison of time to progression and overall survival. a Time to progression between combination therapy and TACE. b Overall survival between combination and TACE. c Overall survival between combination and sorafenib

Several meta-analyses [47-50] and one case-control matched study [51] have reached a consensus that there is a significant improvement of TTP with a combined treatment arm, compared to a TACE-alone arm, but discordance exists with reference to the OS outcomes between them. Of those studies, some results indicated that the use of sorafenib in patients with $\mathrm{HCC}$ concomitantly receiving TACE did not ameliorate the OS compared to those only receiving TACE alone [47-49], whereas Yang et al. [50] pointed out that the OS results 
A

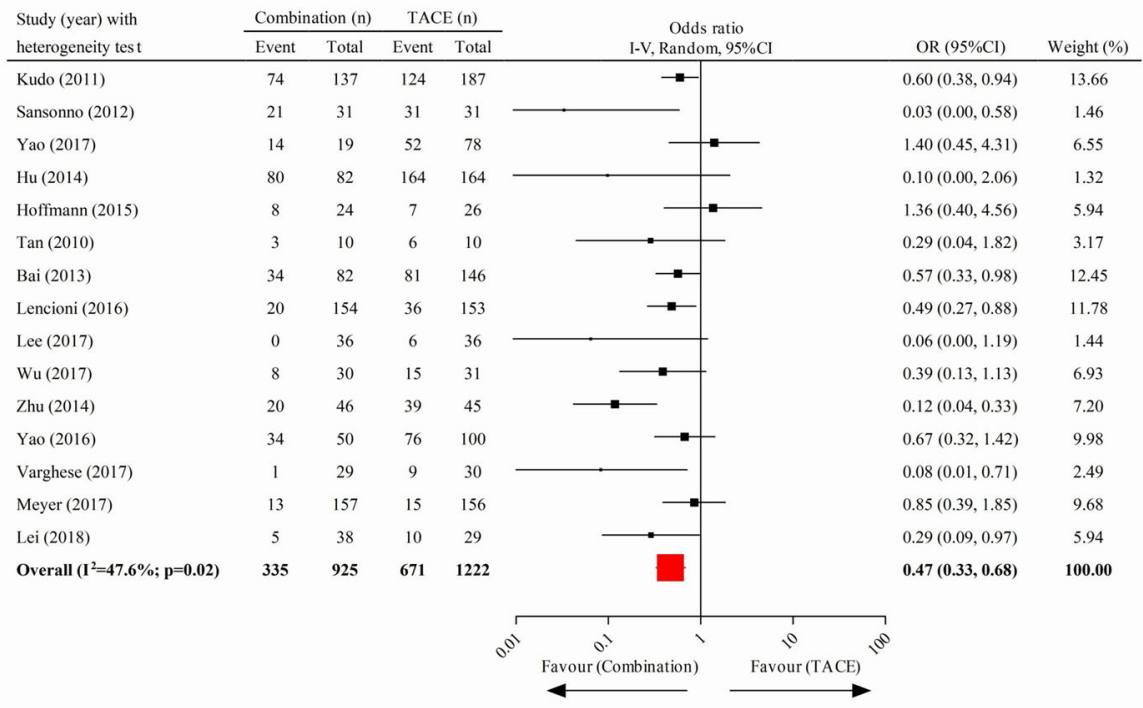

B

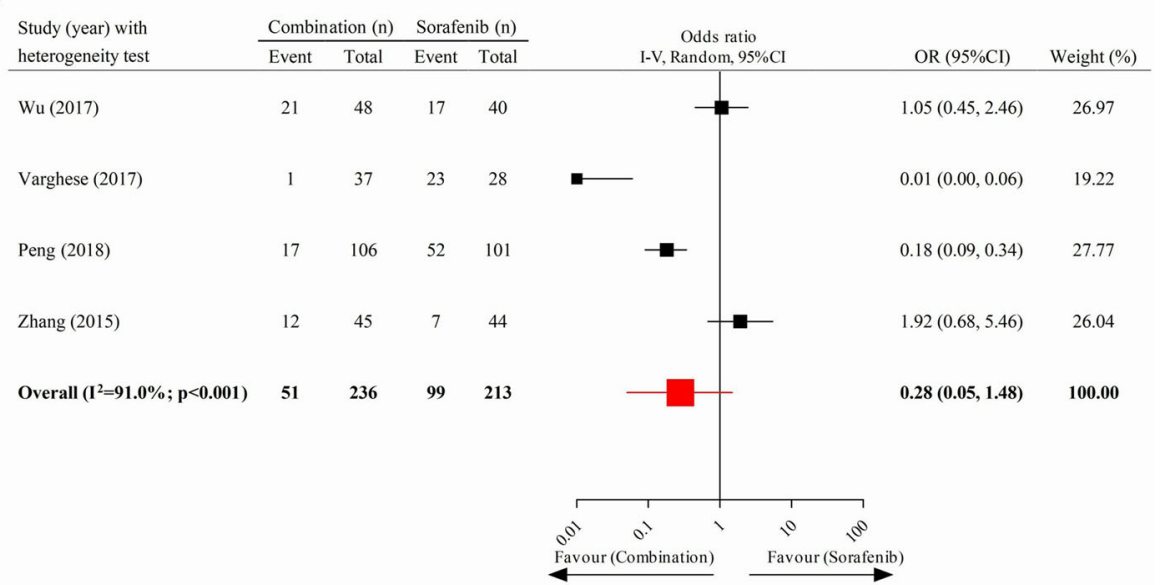

Fig. 3 Comparison of disease progression rate. a Combination therapy vs. TACE. b Combination therapy vs. sorafenib

favoured the combined-treatment group rather than the TACE monotherapy group. The inconsistent conclusions may be because the study by Yang et al. explicitly confines the inclusion criteria to unresectable HCC patients, whereas others did not stipulate this limitation and had fewer analysed subjects. Similarly, two retrospective studies [52, 53] also reported a favourable OS in the combination group as compared to TACE alone.

A systematic review divided patients with advanced HCC based on their region into two subgroups: an Asian countries group and a Western countries group and, interestingly, revealed that the TTP and OS were exclusively prolonged in the Asian countries group but not in the Western countries group after combination therapy, suggesting that the efficacy of combined treatment might be affected by race [54]. Previous meta-analysis showed more improvement in 0.5-year and 1-year survival rates of patients with advanced HCC who underwent combined therapy than those who underwent TACE monotherapy [55]; consistently, our results further support this study, affirming that the 2-year survival rate of those patients was also increased by sorafenib in combination with TACE, even without the diversity of 1-year and 2-year survival rates between the TACE monotherapy cohort and the sorafenib-alone cohort.

Our result, that the OS of patients with advanced HCC treated with combination therapy was superior to that of those patients treated with sorafenib alone, maps to the conclusions of 4 retrospective studies $[19,31,56$, 57] but is in contrast to a clinical trial by Zhang and colleagues in 2015 [43]. In this study, despite a numerically greater median OS in the combined-therapy group than in the sorafenib-alone group (7.3 months vs. 6.0 months), no difference was observed between the two groups $(P=$ 


\section{A}

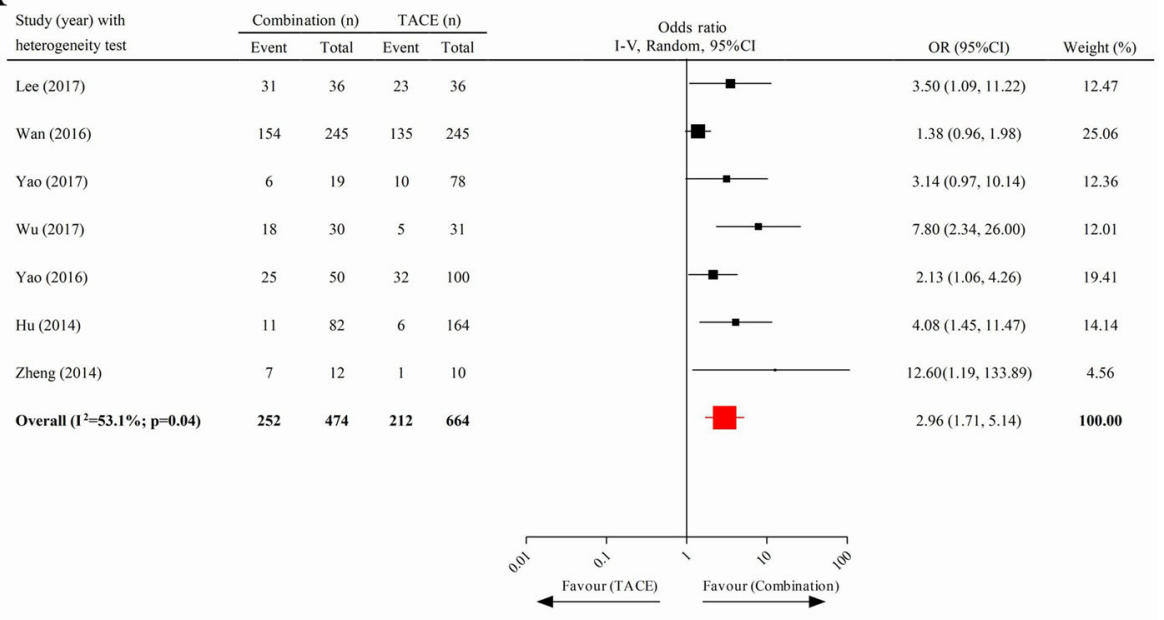

B

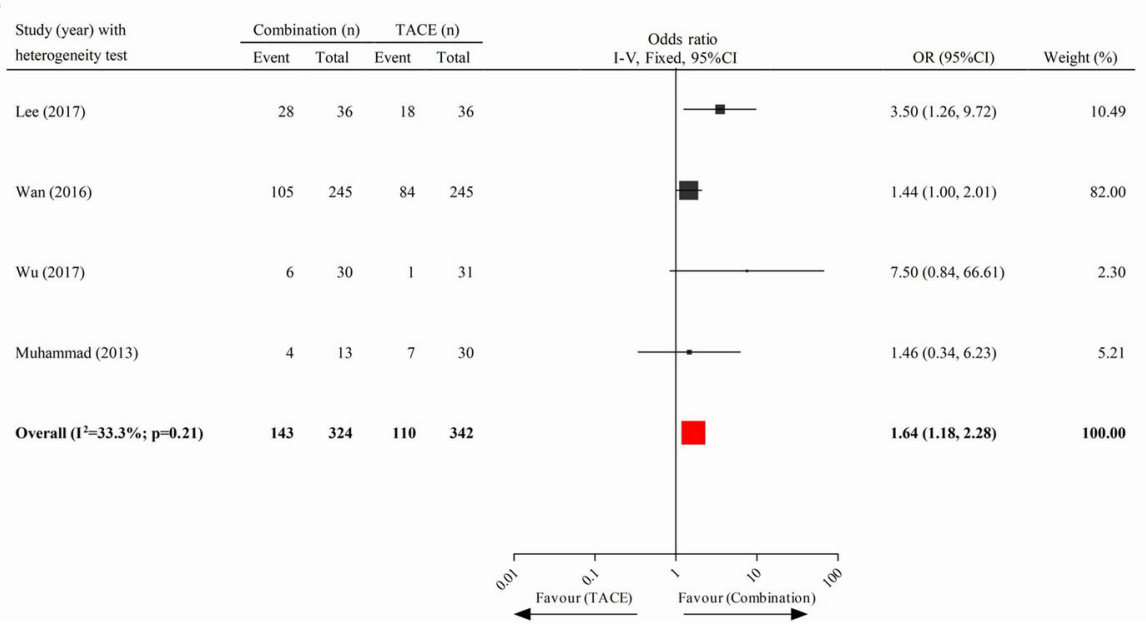

Fig. 4 Analysis of the survival rate between the combination therapy cohort and the TACE cohort. a One-year survival rate. b Two-year survival rate

0.924). The contradiction may be because the enrolled patients in the study by Zhang et al. concurrently had main portal vein tumour thrombosis, which may be an unfavourable factor that affected the efficacy of the combined therapy [14].

Our results mirror the findings from two metaanalyses in which both corroborate the improved tumour regression and disease control of patients with advanced HCC after combination therapy compared to TACE monotherapy [55, 58]; however, the combined treatment may not be superior to TACE alone to increase the ORR and curtail the DPR in patients with early-to-intermediate HCC [59]. In this present analysis, we moreover demonstrate the better ORR in the combination therapy arm than that in the sorafenib-alone arm, but the conclusions of three key clinical trials in this context are contradictory $[19,39,60]$. One explanation is that one of them classified patients with advanced HCC into a BCLC-B stage group and a BCLC$\mathrm{C}$ stage group and demonstrated that the superiority of combination therapy compared to sorafenib monotherapy was manifested only in the former group but not in the latter group, whereas the other studies did not implement this subgroup analysis.

The DPR in the combined-treatment cohort is not greater than that in the sorafenib monotherapy cohort, which may be consistent with the outright opposite effects of expressing the hypoxia-inducible factor- $1 \alpha$ (HIF$1 \alpha)$ and VEGF in patients with advanced HCC undergoing TACE and in those after treatment with sorafenib. First, tumour-feeding arteries are embolised by TACE treatment, inevitably giving rise to the elevation of the HIF-1 $\alpha$ level that is related to tumour recurrence, disease progression and distant metastasis [21, 61]. Second, TACE incites the overexpression of VEGF in HCC, hence promoting angiogenesis [6, 7]. By contrast, 


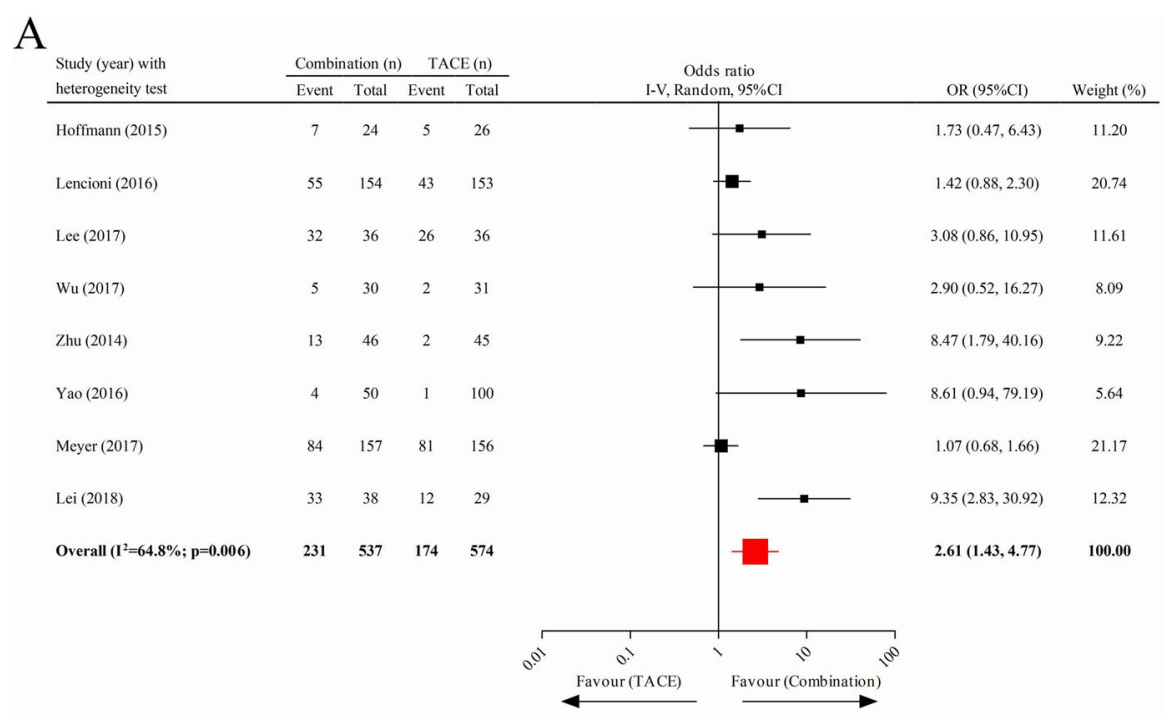

B

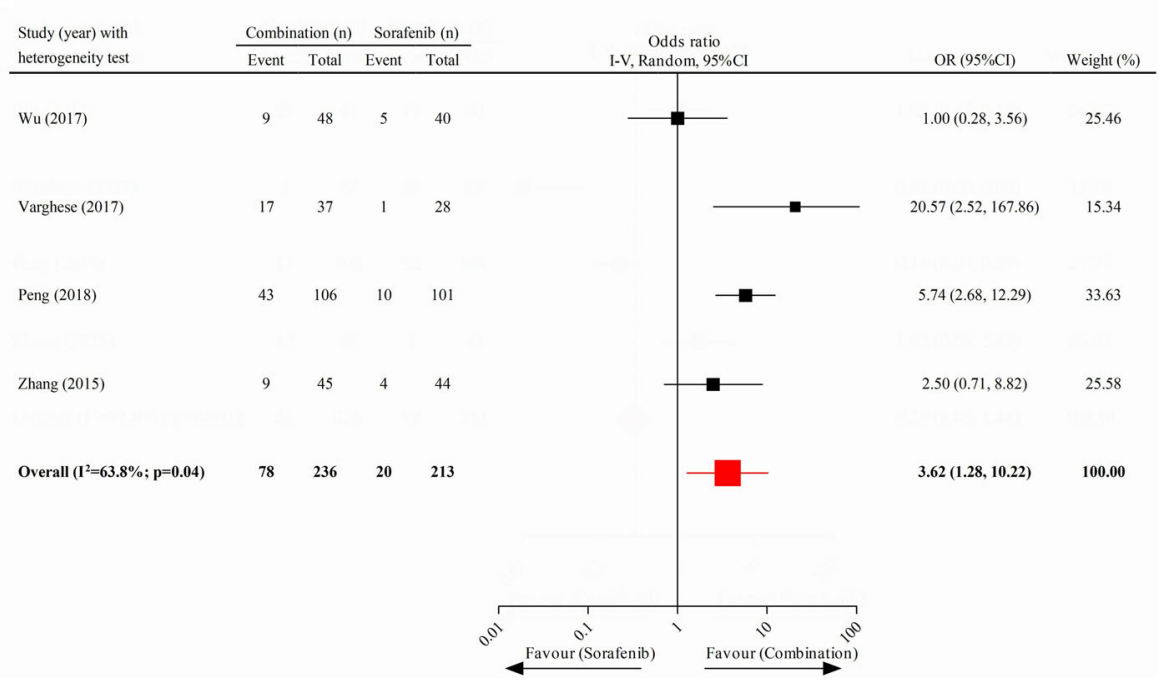

Fig. 5 Comparison of overall response rate. a Combination therapy versus TACE. b Combination therapy versus sorafenib

sorafenib effectively reduces the expression of HIF- $1 \alpha$ and VEGF and inhibits VEGF receptor and plateletderived growth factor receptors, which alleviates the TACE-induced adverse situations [62-64]. Therefore, TACE in conjunction with sorafenib to treat patients with advanced HCC theoretically does not outperform sorafenib alone in decreasing the DPR.

The study has some limitations that deserve special mention. First, including only English language articles might lead to selection bias. Furthermore, only several trials with limited available data were enrolled to conduct some analyses (the DPR of combination therapy versus sorafenib, the 2-year survival rate of combination therapy versus TACE and the ORR of combination therapy versus sorafenib), which might increase the uncertainty of the conclusions. Third, substantial heterogeneity was manifested in almost all analyses, which may be relevant to the differences of study type, treatment procedures and the frequency of tumour assessment. The $P$ value of Egger's test in some analyses also suggested potential publication bias. Additionally, treatment-related adverse events were not assessed in our article because they were tolerable [55]. Last, hepatitis B (HB) virus infection accounts for the predominant reason for HCC, particularly in China, and anti-HB virus therapy can significantly ameliorate HCC patients who house $\mathrm{HB}$ virus; however, there were scanty details documented in these included clinical trials. If it is available to implement a stratified analysis of HCC patients with or without $\mathrm{HB}$ infection, there may be some innovated results. 


\section{Conclusion}

The combination of TACE with sorafenib in treating patients with advanced HCC can prolong TTP and OS, improve ORR and 1-year and 2-year survival rates and reduce the DPR more efficiently than TACE can alone. This combination therapy is also superior to sorafenib monotherapy in terms of the longer OS and higher ORR. As a monotherapy strategy, the 1-year and 2-year survival rates in the TACE arm were identical to those in the sorafenib arm.

\section{Supplementary information}

Supplementary information accompanies this paper at https://doi.org/10 1186/s12957-020-02017-0.

Additional file 1: eFigure 1. The comparison of survival rate between TACE and sorafenib. (A) 1-year survival rate; (B) 2-year survival rate. eTable 1. The publication bias in all analyses. Abbreviations: TTP, time-toprogression; OS, overall survival; DPR, disease progression rate; 1y-SR, 1year survival rate; 2y-SR, 2-year survival rate; ORR, overall response; TACE, transarterial chemoembolization.

\section{Abbreviations}

HCC: Hepatocellular carcinoma; TACE: Transarterial chemoembolisation; OR: Odds ratio; Cl: Confidence interval; SMD: Standardised mean difference; OS: Overall survival; TTP: Time to progression; ORR: Overall response rate; DPR: Disease progression rate; BCLC: Barcelona Clinic Liver Cancer; VEGF: Vascular endothelial growth factor; RECIST: Response Evaluation Criteria in Solid Tumours; mRECIST: Modified Response Evaluation Criteria in Solid Tumours; RCTs: Randomised controlled trials; HIF-1a: Hypoxia-inducible factor-1a

\section{Acknowledgements}

Not applicable.

\section{Authors' contributions}

LZ and ZC conceived the study. ZC, LH and YG conducted the literature search, study selection and data extractions and analysis. ZC, LH, YS and SS drafted the manuscript. LZ, YG, YS and SS contributed in reviewing the manuscript for intellectual content. All authors read and approved the final manuscript.

\section{Funding}

This study did not receive any specific grant from funding agencies in the public, commercial or not-for-profit sectors.

Availability of data and materials

All data are fully available without restriction.

\section{Ethics approval and consent to participate}

All analyses were based on previous published studies; thus, no ethical approval and consent to participate are required.

\section{Consent for publication}

All analyses were based on previous published studies; thus, no consent for publication is required.

\section{Competing interests}

The authors declare that they have no competing interests.

\section{Author details}

${ }^{1}$ Department of Gastroenterology, The Second Hospital of Anhui Medical University, No.678 Furong Road, Jingkai District, Hefei, Anhui Province, China. ${ }^{2}$ Breast Center B ward, The Affiliated Hospital of Qingdao University, Shandong Province, Qingdao, China. ${ }^{3}$ Department of Gastroenterology, The
Affiliated Hospital of Qingdao University, Shandong Province, Qingdao, China.

Received: 27 April 2020 Accepted: 31 August 2020

Published online: 11 September 2020

References

1. Parkin DM. Global cancer statistics in the year 2000. Lancet Oncol. 2001;2(9): 533-43.

2. El-Serag HB, Rudolph KL. Hepatocellular carcinoma: epidemiology and molecular carcinogenesis. Gastroenterology. 2007;132(7):2557-76.

3. Hung H. Treatment modalities for hepatocellular carcinoma. Curr Cancer Drug Targets. 2005;5(2):131-8.

4. Bruix J, Gores GJ, Mazzaferro V. Hepatocellular carcinoma: clinical frontiers and perspectives. Gut. 2014;63(5):844-55.

5. Bruix J, Llovet JM, Castells $A$, et al. Transarterial embolization versus symptomatic treatment in patients with advanced hepatocellular carcinoma: results of a randomized, controlled trial in a single institution. Hepatology. 1998;27(6):1578-83.

6. Bao Y, Feng WM, Tang CW, Zheng YY, Gong HB, Hou EG. Endostatin inhibits angiogenesis in hepatocellular carcinoma after transarterial chemoembolization. Hepato-gastroenterology. 2012;59(117):1566-8.

7. Poon RT, Lau C, Yu WC, Fan ST, Wong J. High serum levels of vascular endothelial growth factor predict poor response to transarterial chemoembolization in hepatocellular carcinoma: a prospective study. Oncol Rep. 2004;11(5):1077-84.

8. Mathonnet M, Descottes B, Valleix D, Labrousse F, Truffinet V, Denizot Y. Quantitative analysis using ELISA of vascular endothelial growth factor and basic fibroblast growth factor in human colorectal cancer, liver metastasis of colorectal cancer and hepatocellular carcinoma. World J Gastroenterol. 2006; 12(23):3782-3.

9. Llovet JM, Ricci S, Mazzaferro V, et al. Sorafenib in advanced hepatocellular carcinoma. N Engl J Med. 2008:359(4):378-90.

10. Wilhelm SM, Carter C, Tang L, et al. BAY 43-9006 exhibits broad spectrum oral antitumor activity and targets the RAF/MEK/ERK pathway and receptor tyrosine kinases involved in tumor progression and angiogenesis. Cancer Res. 2004;64(19):7099-109.

11. Cheng AL, Kang YK, Chen Z, et al. Efficacy and safety of sorafenib in patients in the Asia-Pacific region with advanced hepatocellular carcinoma: a phase III randomised, double-blind, placebo-controlled trial. Lancet Oncol. 2009;10(1):25-34.

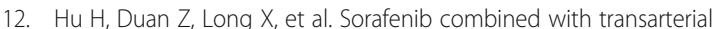
chemoembolization versus transarterial chemoembolization alone for advanced-stage hepatocellular carcinoma: a propensity score matching study. PLoS One. 2014;9(5):e96620.

13. Pan T, Li XS, Xie QK, et al. Safety and efficacy of transarterial chemoembolization plus sorafenib for hepatocellular carcinoma wi th portal venous tumour thrombus. Clin Radiol. 2014;69(12):e553-61.

14. Zhu K, Chen J, Lai L, et al. Hepatocellular carcinoma with portal vein tumor thrombus: treatment with transarterial chemoembolizat ion combined with sorafenib--a retrospective controlled study. Radiology. 2014;272(1):284-93.

15. Stewart LA, Clarke M, Rovers M, et al. Preferred Reporting Items for Systematic Review and Meta-Analyses of individual participant data: the PRISMA-IPD Statement. Jama. 2015;313(16):1657-65.

16. Sutton AJAK, Jones DR. Methods for metaanalysis in medical research. Wiley series in probability and statistics-applied probability and statistics section. Hoboken: Wiley; 2000

17. Jadad AR, Moore RA, Carroll D, et al. Assessing the quality of reports of randomized clinical trials: is blinding necessary? Control Clin Trials. 1996: $17(1): 1-12$.

18. Qu XD, Chen CS, Wang JH, et al. The efficacy of TACE combined sorafenib in advanced stages hepatocellullar carcinoma. BMC Cancer. 2012;12:263.

19. Wu FX, Chen J, Bai T, et al. The safety and efficacy of transarterial chemoembolization combined with sorafenib and sorafenib mono-therapy in patients with $\mathrm{BCLC}$ stage $\mathrm{B} / \mathrm{C}$ hepatocellular carcinoma. BMC Cancer. 2017:17(1):645.

20. Tan WF, Qiu ZQ, Yu Y, et al. Sorafenib extends the survival time of patients with multiple recurrences of hepatocellular carcinoma after liver transplantation. Acta Pharmacol Sin. 2010;31(12):1643-8.

21. Bai W, Wang YJ, Zhao Y, et al. Sorafenib in combination with transarterial chemoembolization improves the survival of patients with unresectable 
hepatocellular carcinoma: a propensity score matching study. J Dig Dis. 2013;14(4):181-90.

22. Lee JM, Jang BK, Lee $Y$ J, et al. Survival outcomes of hepatic resection compared with transarterial chemoembolization or sorafenib for hepatocellular carcinoma with portal vein tumor thrombosis. Clin Mole Hepatol. 2016;22(1):160-7.

23. Nishikawa H, Osaki Y, lguchi E, et al. Comparison of the efficacy of transcatheter arterial chemoembolization and sorafenib for advanced hepatocellular carcinoma. Exp Ther Med. 2012;4(3):381-6.

24. Hoffmann K, Ganten T, Gotthardtp D, et al. Impact of neo-adjuvant sorafenib treatment on liver transplantation in HCC patients - a prospective, randomized, double-blind, phase III trial. BMC Cancer. 2015;15:392.

25. Ren B, Wang W, Shen J, Li W, Ni C, Zhu X. Transarterial chemoembolization (TACE) combined with sorafenib versus TACE alone for unresectable hepatocellular carcinoma: a propensity score matching study. J Cancer. 2019;10(5):1189-96.

26. Kudo M, Imanaka K, Chida N, et al. Phase III study of sorafenib after transarterial chemoembolisation in Japanese and Korean patients with unresectable hepatocellular carcinoma. Eur I cancer (Oxford, England 1990). 2011;47(14):2117-27.

27. Lencioni R, Llovet JM, Han G, et al. Sorafenib or placebo plus TACE with doxorubicin-eluting beads for intermediate stage HCC: the SPACE trial. J Hepatol. 2016;64(5):1090-8

28. Arizumi T, Ueshima $\mathrm{K}$, Minami $T$, et al. Effectiveness of sorafenib in patients with transcatheter arterial chemoembolization (TACE) refractory and intermediate-stage hepatocellular carcinoma. Liver Cancer. 2015;4(4):253-62.

29. Lee $T Y$, Lin CC, Chen CY, et al. Combination of transcatheter arterial chemoembolization and interrupted dosing sorafenib improves patient survival in early-intermediate stage hepatocellular carcinoma. Medicine (United States). 2017:96(37):e7655.

30. Sansonno D, Lauletta G, Russi S, Conteduca V, Sansonno L, Dammacco F. Transarterial chemoembolization plus sorafenib: a sequential therapeutic scheme for HCV-related intermediate-stage hepatocellular carcinoma: a randomized clinical trial. BMC Gastroenterol. 2012;17(3):359-66.

31. Ha Y, Lee D, Shim JH, et al. Role of transarterial chemoembolization in relation with sorafenib for patients with advanced hepatocellular carcinoma. Oncotarget. 2016;7(45):74303-13.

32. Wan $X$, Zhai $X$, Yan Z, et al. Retrospective analysis of transarterial chemoembolization and sorafenib in Chinese patients with unresectable and recurrent hepatocellular carcinoma. Oncotarget. 2016;7(50):8380616.

33. Yao $Q$, Zhang $H$, Xiong B, Zheng C. Combination of sorafenib and TACE inhibits portal vein invasion for intermediate stage HCC: a single center retrospective controlled study. Oncotarget. 2017;8(45):79012-22.

34. Wu J, Li A, Yang J, Lu Y, Li J. Efficacy and safety of TACE in combination with sorafenib for the treatment of TACE-refractory advanced hepatocellular carcinoma in Chinese patients: a retrospective study. Onco Targets Therapy. 2017;10:2761-8

35. Meyer T, Fox R, Ma YT, et al. Sorafenib in combination with transarterial chemoembolisation in patients with unresectable hepatocellular carcinoma (TACE 2): a randomised placebo-controlled, double-blind, phase 3 trial. Lancet Gastroenterol Hepatol. 2017;2(8):565-75.

36. Ogasawara S, Chiba T, Ooka Y, et al. Efficacy of sorafenib in intermediatestage hepatocellular carcinoma patients refractory to transarterial chemoembolization. Oncology. 2014;87(6):330-41.

37. Yao X, Yan D, Zeng H, Liu D, Li H. Concurrent sorafenib therapy extends the interval to subsequent TACE for patients with unresectable hepatocellular carcinoma. J Surg Oncol. 2016;113(6):672-7.

38. Zhao Y, Li H, Bai W, et al. Early sorafenib-related adverse events predict therapy response of TACE plus sorafenib: a multicenter clinical study of 606 HCC patients. Int J Cancer. 2016;139(4):928-37.

39. Varghese J, Kedarisetty CK, Venkataraman J, et al. Combination of TACE and sorafenib improves outcomes in BCLC stages B/C of hepatocellular carcinoma: a single centre experience. Ann Hepatol. 2017;16(2):247-54.

40. Zhu K, Huang J, Lai L, et al. Medium or large hepatocellular carcinoma: sorafenib combined with transarterial chemoembolization and radiofrequency ablation. Radiology. 2018;288(1):300-7.

41. Peng Z, Chen S, Wei M, et al. Advanced recurrent hepatocellular carcinoma: treatment with sorafenib alone or in combination with transarterial chemoembolization and radiofrequency ablation. Radiology. 2018;287(2): 705-14.
42. Pinter M, Hucke F, Graziadei I, et al. Advanced-stage hepatocellular carcinoma: transarterial chemoembolization versus sorafenib. Radiology. 2012;263(2):590-9.

43. Zhang Y, Fan W, Wang Y, et al. Sorafenib with and without transarterial chemoembolization for advanced hepatocellular carcinoma with main portal vein tumor thrombosis: a retrospective analysis. Oncologist. 2015; 20(12):1417-24

44. Lei XF, Ke Y, Bao TH, et al. Effect and safety of sorafenib in patients with intermediate hepatocellular carcinoma who received transarterial chemoembolization: a retrospective comparative study. World J Clin Cases. 2018;6(5):74-83.

45. Zheng SZ, Liu DJ, Sun P, et al. Feasibility and safety of sorafenib treatment in hepatocellular carcinoma patients with spontaneous rupture. World J Gastroenterol. 2014;20(43):16275-81.

46. Muhammad A, Dhamija M, Vidyarthi G, et al. Comparative effectiveness of traditional chemoembolization with or without sorafenib for hepatocellular carcinoma. World J Hepatol. 2013;5(7):364-71.

47. Li J, Liu W, Zhu W, Wu Y, Wu B. Transcatheter hepatic arterial chemoembolization and sorafenib for hepatocellular carcinoma: a metaanalysis of randomized, double-blind controlled trials. Oncotarget. 2017; 8(35):59601-8

48. Wang G, Liu Y, Zhou SF, et al. Sorafenib combined with transarterial chemoembolization in patients with hepatocellular carcinoma: a metaanalysis and systematic review. Hepatol Int. 2016;10(3):501-10.

49. Hu MD, Jia LH, Liu HB, Zhang KH, Guo GH. Sorafenib in combination with transarterial chemoembolization for hepatocellular carcinoma: a metaanalysis. Eur Rev Med Pharmacol Sci. 2016;20(1):64-74.

50. Yang M, Yuan JQ, Bai M, Han GH. Transarterial chemoembolization combined with sorafenib for unresectable hepatocellular carcinoma: a systematic review and meta-analysis. Mol Biol Rep. 2014;41(10):6575-82.

51. Zhang YF, Wei W, Wang JH, et al. Transarterial chemoembolization combined with sorafenib for the treatment of hepatocellular carcinoma with hepatic vein tumor thrombus. OncoTargets Therapy. 2016;9:4239-46.

52. Liu Q, Dai Y. Sorafenib combined with transarterial chemoembolization prolongs survival of patients with advanced hepatocellular carcinoma. J BUON. 2020;25(2):945-51.

53. Lin PT, Teng W, Jeng WJ, et al. Add-on sorafenib is beneficial for hepatocellular carcinoma patients with transarterial chemoembolization refractoriness: a real-world experience. Eur J Gastroenterol Hepatol. 2020; 32(9):1192-9.

54. Li L, Zhao W, Wang M, et al. Transarterial chemoembolization plus sorafenib for the management of unresectable hepatocellular carcinoma: a systematic review and meta-analysis. BMC Gastroenterol. 2018;18(1):138.

55. Cai R, Song R, Pang P, et al. Transcatheter arterial chemoembolization plus sorafenib versus transcatheter arterial chemoembolization alone to treat advanced hepatocellular carcinoma: a meta-analysis. BMC Cancer. 2017; 17(1):714.

56. Kok VC, Chen YC, Chen YY, Su YC. Sorafenib with transarterial chemoembolization achieves improved survival vs. sorafenib alone in advanced hepatocellular carcinoma: a nationwide population-based cohort study. Cancers (Basel). 2019;11(7):985.

57. Chien SC, Chen CY, Cheng PN, et al. Combined transarterial embolization/ chemoembolization-based locoregional treatment with sorafenib prolongs the survival in patients with advanced hepatocellular carcinoma and preserved liver function: a propensity score matching study. Liver Cancer. 2019;8(3):186-202.

58. Zhang $X$, Wang $K$, Wang $M$, et al. Transarterial chemoembolization (TACE) combined with sorafenib versus TACE for hepatocellular carcinoma with portal vein tumor thrombus: a systematic review and meta-analysis. Oncotarget. 2017;8(17):29416-27.

59. Zeng J, Lv L, Mei ZC. Efficacy and safety of transarterial chemoembolization plus sorafenib for early or intermediate stage hepatocellular carcinoma: a systematic review and meta-analysis of randomized controlled trials. Clin Res Hepatol Gastroenterol. 2016;40(6):688-97.

60. Park JW, Kim YJ, Kim DY, et al. Sorafenib with or without concurrent transarterial chemoembolization in patients with advanced hepatocellular carcinoma: the phase III STAH trial. J Hepatol. 2019;70(4):684-91.

61. Wada H, Nagano H, Yamamoto $H$, et al. Expression pattern of angiogenic factors and prognosis after hepatic resection in hepatocellular carcinoma: importance of angiopoietin-2 and hypoxia-induced factor-1 alpha. Liver Int. 2006;26(4):414-23. 
62. Scartozzi M, Faloppi L, Svegliati Baroni G, et al. VEGF and VEGFR genotyping in the prediction of clinical outcome for HCC patients receiving sorafenib: the ALICE-1 study. Int I Cancer. 2014;135(5):1247-56.

63. Villanueva A, Llovet JM. Targeted therapies for hepatocellular carcinoma. Gastroenterology. 2011;140(5):1410-26.

64. Ma L, Li G, Zhu H, et al. 2-Methoxyestradiol synergizes with sorafenib to suppress hepatocellular carcinoma by simultaneously $\mathrm{d}$ ysregulating hypoxia-inducible factor-1 and -2. Cancer Lett. 2014;355(1):96-105.

\section{Publisher's Note}

Springer Nature remains neutral with regard to jurisdictional claims in published maps and institutional affiliations.

Ready to submit your research? Choose BMC and benefit from:

- fast, convenient online submission

- thorough peer review by experienced researchers in your field

- rapid publication on acceptance

- support for research data, including large and complex data types

- gold Open Access which fosters wider collaboration and increased citations

- maximum visibility for your research: over $100 \mathrm{M}$ website views per year

At $\mathrm{BMC}$, research is always in progress.

Learn more biomedcentral.com/submissions 